\title{
Mermithid nematodes found in adult Anopheles from southeastern Senegal
}

\author{
Kevin C Kobylinski*, Massamba Sylla, William Black IV and Brian D Foy
}

\begin{abstract}
Background: Over two dozen mermithid nematodes have been described parasitizing mosquitoes worldwide, however, only two species were found in Africa. Mermithid nematodes kill their mosquito host upon emergence, which suggests that they could be developed as biological control agents of mosquitoes. Both Romanomermis culicivorax and Romanomermis iyengari have been reared for mass release to control numerous Anopheles species vector populations, and in one instance this may have led to reduced malaria prevalence in a human population.

Methods: Anopheles mosquitoes were collected during a malaria study in southeastern Senegal. Two different adult blood fed mosquitoes had a single mermithid nematode emerge from their anus while they were being held post-capture. Primers from the $18 \mathrm{~S} \mathrm{rDNA}$ were developed to sequence nematode DNA and screen mosquitoes for mermithid DNA. $18 \mathrm{~S}$ rDNA from the Senegalese mermithid and other mermithid entries in GenBank were used to create a Maximum Parsimony tree of the Mermithidae family.

Results: The mermithid was present in 1.8\% (10/551) of the sampled adult Anopheles species in our study area. The mermithid was found in An. gambiae s.s., An. funestus, and An. rufipes from the villages of Ndebou, Boundoucondi, and Damboucoye. Maximum parsimony analysis confirmed that the nematode parasites found in Anopheles were indeed mermithid parasites, and of the mermithid sequences available in GenBank, they are most closely related to Strelkovimermis spiculatus.

Conclusions: To our knowledge, this is the first report of mermithids from adult Anopheles mosquitoes in Senegal. The mermithid appears to infect Anopheles mosquitoes that develop in diverse larval habitats. Although maximum parsimony analysis determined the mermithid was closely related to Strelkovimermis spiculatus, several characteristics of the mermithid were more similar to the Empidomermis genus. Future mermithid isolations will hopefully allow: formal taxonomic identification, laboratory colonization, determination of life history traits and species specificity, and characterize its usefulness as a biological control agent.
\end{abstract}

Keywords: Mermithidae, Anopheles, Senegal

\section{Background}

Nematodes from the family Mermithidae (Enoplea: Mermithida) parasitize terrestrial and aquatic invertebrates, including many insect disease vector species [1]. Many insect disease vectors are parasitized by mermithid nematodes during their aquatic larval stage, including blackflies and mosquitoes [2]. Seven genera of Mer mithidae are parasites of mosquitoes: Culicimermis, Empi domermis, Hydromermis, Octomyomermis, Perutilimermis,

\footnotetext{
*Correspondence: kobylinskikevin@yahoo.com

Arthropod-borne Infectious Disease Laboratory, Department of Microbiology, Immunology and Pathology, Colorado State University, 1692 Campus Delivery, Fort Collins, CO 80523-1692, USA
}

Romanomermis, and Strelkovimermis [3], and over 25 species have been described [4].

While numerous mermithids have been found in mosquitoes on every continent except Antarctica [4-8], only Empidomermis cozii and Reesimermis (Octomyomermis) muspratti, have been described from mosquitoes in Africa [9-11]. Octomyomermis muspratti was isolated from various Aedes and Culex spp. larvae from tree holes in Livingstone, Zambia [11]. Subsequent colonies were established and O. muspratti was found to be capable of parasitizing Aedes aegypti, Aedes polynesiensis, Culex pipiens, Anopheles stephensi, and Anopheles

\section{Biomed Central}


albimanus [12]. Empidomermis cozii was found in adult Anopheles funestus from Burkina Faso $[9,10]$.

Regardless of genera, aquatic mermithids that infect mosquitoes have similar life cycles. Eggs hatch in either standing or flowing water and the pre-parasitic forms attach to and bore into mosquito larvae. Parasitism is typically most successful when the pre-parasitic form invades a first or second instar mosquito. Pre-parasitic mermithids develop in the mosquito host, molt once, and emerge from the mosquito as post-parasitic forms. Mermithid emergence is typically lethal for the mosquito and may happen in the mosquito larval or adult stage. In the aquatic habitat the post-parasitic mermithids molt into adults, mate and lay eggs. The development time of each mermithid life stage varies among species, and this dictates at which mosquito life stage the post-parasitic form emerges [13].

Mosquito death upon mermithid emergence has sparked interest in using mermithids as biological control agents. From a classical biological control standpoint, Juliano (2007) cited several characteristics of mermithids that make them potentially successful biological control agents of mosquitoes: they are specialists, they develop in synchrony with mosquitoes, their populations can increase rapidly or be augmented by human reinundation, and they require only one victim/host to complete their life cycle [14]. Platzer (2007) summarizes multiple characteristics of mermithids that make them attractive for biological control of mosquitoes, including ease of application, environmental safety, host specificity, laboratory manipulation of life history, lethality, mass rearing in vivo, and potential for long-term recycling in the environment. Numerous investigators have developed mass rearing techniques for mermithids [15-17] that can support large scale field releases. Mermithids have been applied to aquatic habitats as either pre-parasitic forms with a hand operated spray pump [17] or aerial spray pump [18] and post-parasitic forms applied to moist soil prior to flooding $[19,20]$.

Major field releases of both Romanomermis culicivorax and Romanomermis iyengari have occurred for Anopheles population control. Romanomermis iyengari has been used to suppress malaria vector populations of $A n$. albimanus in Cuba [21], Anopheles pseudopunctipennis in Oaxaca State, Mexico [22], and Anopheles albitarsis and Anopheles rondoni in Roraima State, Brazil [23]. Romanomermis culicivorax has been used to suppress malaria vector populations of Anopheles freeborni in California, USA [18,19], An. albimanus and An. pseudopunctipennis in El Salvador [16,24], An. albimanus in Mella Isla de la Juventud, Cuba [25], and An. albimanus in El Valle, Colombia [26]. The publication by Rojas et al. (1987) is notable because release of $R$. culicivorax may have both suppressed the vector population and reduced malaria prevalence in local children for up to two years post mermithid release. The successful control of An. albimanus by $R$. culicivorax, a mermithid that exits the mosquito in the larval stage, was facilitated by the fact that $A n$. albimanus larvae occur in large standing bodies of water that can be readily identified and treated with mermithids [26]. This evidence demonstrates that mermithid nematodes can be successfully used as biological control agents of mosquitoes.

This report documents numerous mermithid nematodes that were observed in adult female Anopheles spp. collected during a malaria study conducted in southeastern Senegal [27]. Sequencing and phylogeny analysis were used to place the nematodes in the Mermithidae family and compare their relatedness to other mermithid species.

\section{Methods}

All mosquitoes were collected in 2009 from the villages of Ndebou, Boundoucondi, Nathia, and Damboucoye in southeastern Senegal [28]. Blood fed mosquitoes were collected from huts during the mornings by backpack aspiration and held in a field insectary for up to five days [27]. Other mosquitoes were collected from CDC light traps hung next to bed nets; these mosquitoes were processed on the same day of capture. Mosquitoes were identified morphologically [29] and molecularly [30,31] to species. During routine morphological examination, mosquitoes were also inspected visually for possible mermithid infection. Adult mermithids range in length from 10 to $100 \mathrm{~mm}$ [1] so they can be readily observed without dissection of the mosquito (see Figure 1a). Mermithid nematodes were only observed in mosquito abdomens. Abdomens were separated from the thorax of the adult mosquitoes and stored on silica gel desiccant.

Two mermithid-positive $A n$. gambiae s.s. were dissected and the nematodes were separated from the mosquitoes so that mermithid DNA could be extracted with the Qiagen DNeasy kit (Qiagen Sciences, Maryland, USA) and a Qiacube robot (Qiagen Sciences, Maryland, USA). Primers were designed to amplify regions of the $18 \mathrm{~S}$ ribosomal RNA gene by the polymerase chain reaction (PCR). Primers were designed by alignment of sequences from other mermithids obtained from GenBank and making consensus primers. Primer sequences were: Merm forward - 5'-CAAGGACGAAAGTTAGAGGTTC-3' and Merm reverse - 5'-GGAAACCTTGTTACGACTTTTA-3'. Twenty $\mu$ of a PCR enzyme mixture was used to amplify DNA from each sample; this mixture consisted of: $10 \mu$ l Fermentas 2X Master Mix, $2 \mu$ l forward and reverse primers at $10 \mu \mathrm{M}, 2 \mu \mathrm{l}$ DNA, and $4 \mu \mathrm{l}$ of RNAse-free water. The thermocycler protocol was 5 minutes at $95^{\circ} \mathrm{C}, 30$ seconds denaturing at $95^{\circ} \mathrm{C}$, 30 seconds annealing at $50^{\circ} \mathrm{C}$, and 45 seconds extension at 

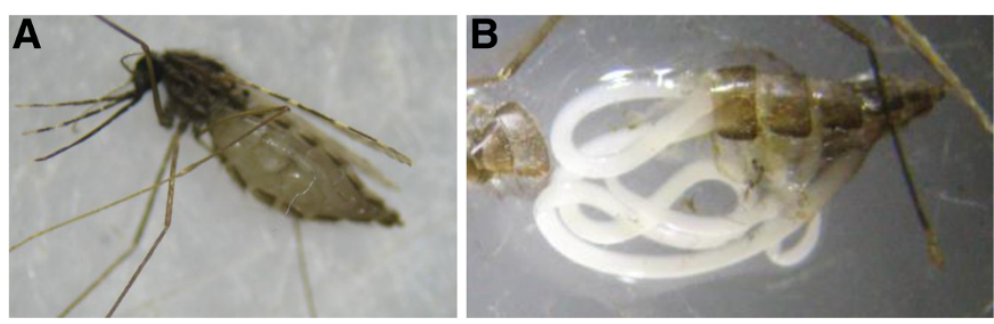

Figure 1 Photograph of a mermithid-parasitized An. rufipes (A) and the dissected mosquito abdomen with mermithid parasite (B).

$72^{\circ} \mathrm{C}$ for 40 cycles with an additional 10 minute extension step, all performed on a PTC-200 Peltier Thermal Cycler (M) Research, Waltham, MA, USA). This protocol was used to assess the presence of mermithid DNA from the abdomens of blood fed mosquitoes that were processed for blood meal analysis [27]. The primers amplified an 804 base pair fragment of mermithid DNA and also a 900 base pair fragment of Anopheles spp. DNA. Thus, when these primers were used to screen wild Anopheles mosquitoes one band would always be present when a mosquito was processed, but when the mosquito was infected with a mermithid parasite, there would be two bands present (see Figure 2).

$18 \mathrm{~S}$ rDNA amplicons from mermithid-only isolates were cloned in a TOPO-TA plasmid (Invitrogen, Carlsbad, CA, USA), plasmid DNA was harvested from transformed E. coli with a QIAprep Spin Miniprep Kit (Qiagen, Gaithersburg, Maryland, USA) and DNA was submitted for sequencing by the Proteomics and Metabolomics Facility at Colorado State University, Fort Collins, Colorado.

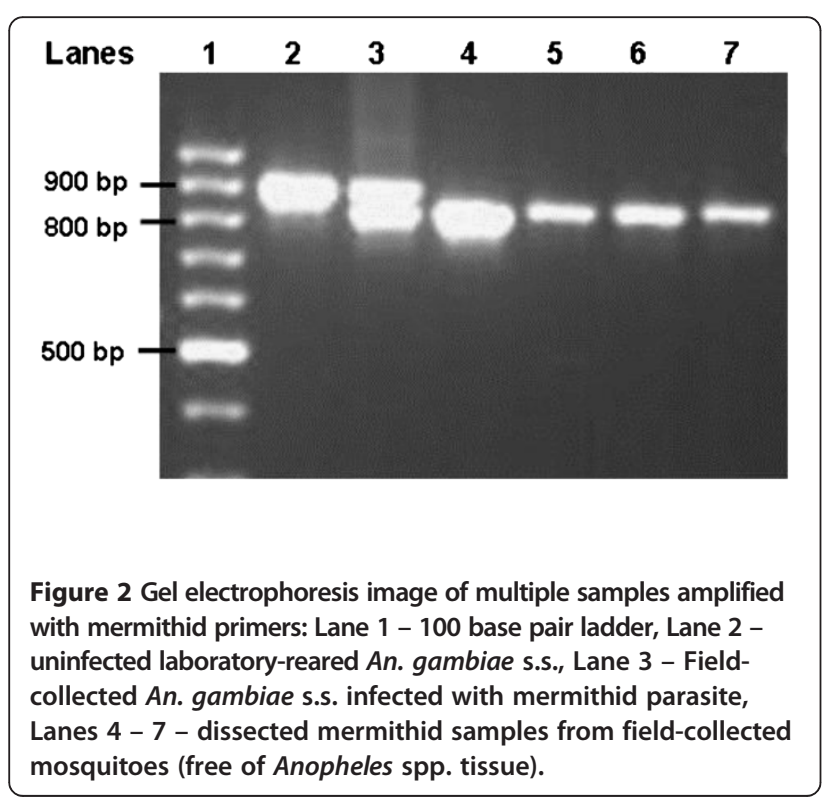

Sequenced mermithid DNA was then used to identify 147 of the most similar sequences published in GenBank, based on E-score. Sequences were provisionally aligned using Clustal W [32]. This alignment was further adjusted by hand, based on the current understanding of RNA secondary models $[33,34]$. Maximum parsimony (MP) phylogenetic analysis was performed using the Phylogenetic Analysis Using Parsimony (PAUP) 4.0b10 package [35]. MP was chosen because it is the only phylogenetic method that uses gaps as phylogenetically informative characters in sequence alignments and gaps were common in the $18 \mathrm{~S}$ ribosomal RNA alignments. Four levels of outgrouping with 100 rounds of bootstrapping were performed. Nematodes from the Mermithidae family formed a monophyletic clade and therefore only sequences from these were compared for the final tree [GenBank Accession Nos.: Agamermis changshaensis DQ628908.1, Agamermis sp. BH-2006 - DQ665653.1, Agamermis xianyangensis - EF617352.1, Allomermis solenopsii - DQ533953.1, Amphimermis sp. A-2007 EF617354.1, Amphimermis sp. B-2007 - EF617355.1, Cryptonchus tristis - EF207244.1, Dintheria tenuissima AY593942.1, Gastromermis sp. - AY146543.1, Gastromermis sp. BH-2006 - DQ533954.1, Heleidomermis sp. BH-2006 - DQ533955.1, Hexamermis agrotis - DQ530 350.1, Isomermis lairdi - FN400900.1, Mermis nigrescens AF036641.1, Mermithid sp. Jh-2004 - AY284743.1, Mermithidae sp. A-AV-2003 - AY374415.1, Mermithidae sp. C-AV-2003 - AY374417.1, Mermithidae sp. MHMH-2008 - FJ040480.1, Octomyomermis huazangensis - EF617353.1, Ovomermis sinensis - DQ520879.1, Romanomermis culicivorax - DQ418791.1, Romanomermis sichuanensis - EF612769.1, Romanomermis wuchangensis - DQ520878.1, Strelkovimermis spiculatus DQ665654.1, Thalassoalaimus pirum - FJ040500.1, and Thaumamermis cosgrovei - DQ665655.1].

\section{Results}

Mermithid DNA was amplified in a total of ten of 551 (1.8\%) Anopheles spp. abdomens collected from 
southeastern Senegal. Mermithids were visually observed in four of these captured Anopheles spp. mosquitoes, and six others were detected only by PCR. Two of the four mermithid nematodes observed visually had emerged from the anus of aspirated mosquitoes that died during the five day holding period, similar to the description and photograph of E. cozii in Poinar (1977). One An. rufipes that had survived to five days post capture contained a live mermithid (Figure 1), but attempts to colonize the mermithid failed. Mermithid-positive Anopheles spp. were found from 3 of the 4 villages from which we sampled mosquitoes: Ndebou (5), Boundoucondi (3), and Damboucoye (2). Five of 74 (6.8\%) An. gambiae s.s. tested from Ndebou, two of 91 (2.2\%) An. funestus and one An. rufipes tested from Boundoucondi, and two of 51 (3.9\%) An. funestus tested from Damboucoye were positive for mermithid parasites or mermithid DNA.

Maximum parsimony analysis confirmed that the nematode parasites found in Anopheles from Senegal were indeed mermithid parasites. A clade containing Senegalese mermithids and Strelkovimermis spiculatus had $72 \%$ bootstrap support (Figure 3 ).

\section{Discussion}

To our knowledge, this is the first report of a mermithid nematode from Anopheles mosquitoes in Senegal. The MP bootstrap analysis placed the Senegalese mermithid in the same clade with Strelkovimermis spiculatus. There are no Empidomermis, Culicimermis, Hydromermis, or
Perutilimermis $18 \mathrm{~S}$ rDNA entries in GenBank, therefore the exact placement of the Senegalese mermithid within the family Mermithidae is unknown. The two previous mermithid species isolated from Africa, E. cozii $[9,10]$ and O. muspratti $[11,12]$ do not have published $18 \mathrm{~S}$ ribosomal RNA sequences. The distance of the Senegalese mermithid from Octomyomermis huazangensis in the MP tree (Figure 3) suggests that this parasite does not belong to the Octomyomermis genera. It is possible that the Senegalese mermithids were re-isolations of $E$. cozii or a different mermithid species not listed in GenBank and therefore not a novel mermithid parasite isolation. The Merm primers developed here could be useful for screening mosquito populations for novel mermithid isolates. Re-isolation of the Senegalese mermithid and proper preparation of specimens for taxonomic evaluation will be necessary to make a more accurate species identification.

Only two Strelkovimermis species have been identified as parasites of mosquitoes, Strelkovimermis spiculatus [8] and Strelkovimermis peterseni [36]. Both S. spiculatus [37] and S. peterseni [36] have only been reported to exit the mosquito in the larval stage. Strelkovimermis spiculatus parasitize Anopheles species in laboratory settings but not as efficiently as Culex and Aedes species [37,38]. Conversely, S. peterseni was only capable of parasitizing Anopheles species larvae and not any of the Culex, Aedes, or Psorophora larvae tested [39]. Similar to other mermithid species, non-target aquatic arthropods

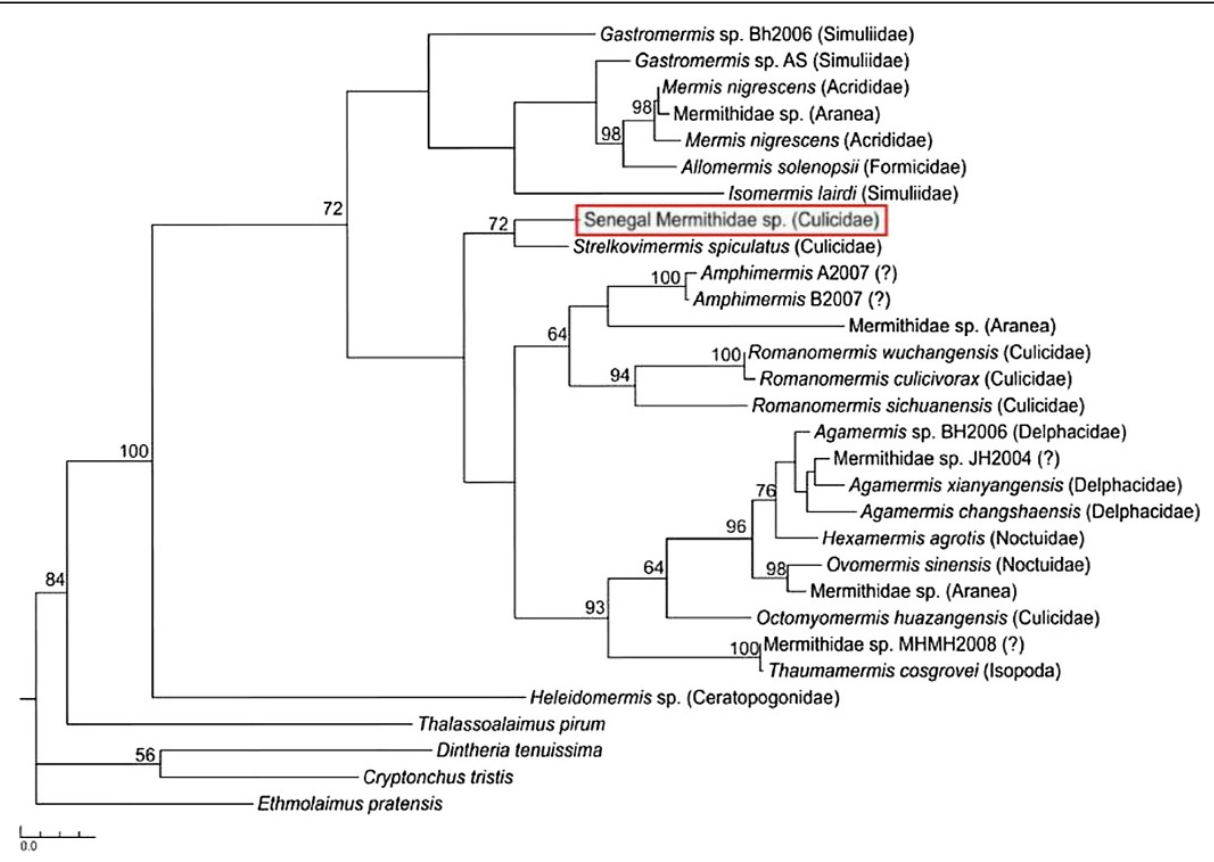

Figure 3 The Maximum Parsimony tree demonstrates that the nematode found in various Anopheles spp. from Senegal is indeed a mermithid parasite. Numbers at nodes represent the degree of bootstrap support. The scale represents the number of steps. Mermithid species names in italics are followed by the family or order the parasite was isolated from in parentheses. 
were rarely parasitized by $S$. spiculatus, and therefore Strelkovimermis species may be desirable for use as a biological control agent $[37,38]$. To date, no field release of either S. spiculatus or S. peterseni has occurred [13].

The Senegalese mermithid may have life cycle characteristics similar to Empidomermis spp. Three isolations of Empidomermis spp. have been documented: E. cozii from Burkina Faso [9,10], Empidomermis riouxi from France [40], and Empidomermis spp. from New York, USA [41]. Empidomermis cozii was reported to emerge from the anus of adult mosquitoes [10], similar to what we observed with the Senegalese mermithid. Empidomermis cozii cannot fully develop in the adult mosquito unless a blood meal has been ingested by the mosquito [10], and all of the mermithid-positive mosquitoes during our study were blood-fed at the point of capture.

Senegalese mermithids were observed or detected in roughly $2 \%$ of the Anopheles in our study area which demonstrates that mermithids are naturally present in Anopheles populations in West Africa. The Merm primers amplified both mosquito and nematode DNA, therefore sequence data was only available for mermithids that had emerged from the adult mosquito and it is not possible to tell if all mermithids detected were the same species. Mermithid parasites were found in An. gambiae s.s., An. funestus, and An. rufipes. If these mermithid specimens were the same nematode species in all three mosquito species, this would indicate that the mermithid has a broad host range within the Anopheles genera. In the future, additional sequencing will be necessary to determine if the Senegalese mermithids from all infected Anopheles species are the same mermithid species or possibly distinct molecular variants as was observed with Mesomermis flumenalis in multiple Simulium species [42]. Anopheles gambiae s.s., An. funestus, and $A n$. rufipes inhabit a diverse and disparate range of larval habitats with typically little overlap [43], therefore the transmission of the Senegalese mermithid may not be restricted by larval habitat type. A mermithid with a broad mosquito host range is more likely to be recycled in the environment which would reduce the number of releases necessary for biological control of mosquito populations [13].

Several characteristics of the development of Empido mermis and the Senegalese mermithid described here may make it an ideal biological control agent for the African malaria vector Anopheles gambiae. Larval control of Anopheles gambiae is difficult because its larval habitats are extremely abundant, ephemeral or inaccessible [44]. A mermithid parasite that emerges from the adult could be dispersed to cryptic An. gambiae larval habitats. Biological control of mosquito larvae in ephemeral larval habitats is difficult due to issues of larval density dependence, compensatory mortality, and overcompensation [14]; however, a mermithid that emerges from the adult mosquito would bypass these issues of biological control. Furthermore, mermithid parasites that emerge from the adult mosquito may be more effective for vector-borne disease control than a mermithid that emerges from a larval mosquito as this is likely to reduce the daily probability of adult survival, the most critical variable in the vectorial capacity equation [45]. It was speculated that Isomermis lairdi, a mermithid parasite of Simulium damnosum that emerges in the adult life stage of the black fly, may limit Onchocerca volvulus transmission by killing the adult black fly vector before $O$. volvulus could fully develop and be transmitted [46]. All three Empidomermis species were reported to sterilize adult mosquitoes [9,40,41]. Empidomermisparasitized females had arrested ovarian development $[40,41]$ and inhibited mating activity of parasitized males [40]. These characteristics would maximize transmission of the mermithid to other mosquito offspring as opposed to infection of the parasitized females own offspring. For ideal mermithid-based control of malaria parasite transmission, the mermithid would need to emerge from the adult mosquito prior to Plasmodium completion of the extrinsic incubation period in the Anopheles vector. There is also the possibility that the mermithid may inhibit Plasmodium development in the mosquito, which could further impact vector-borne disease control. While a mermithid that emerges from the mosquito adult stage may be more difficult to rear in large numbers for mass release, this same life history trait may be used to facilitate a novel, rapid dispersal method of the mermithid into the environment if mermithid-infected adult mosquitoes were released.

The re-isolation of the Senegalese mermithid from adult mosquitoes should be feasible. Specimens should be prepared and submitted to a nematode taxonomist to make a formal identification and description of the species. Laboratory colonies should be established that utilize rearing techniques published for several other mermithids from mosquitoes [15,17]. Sampling of wild adult and larval mosquito populations irrespective of mosquito genera over a longer duration of time combined with DNA barcoding of mermithids will determine critical information on host and temporal range of the mermithid. The life cycle and interactions of the Senegalese mermithid with various Anopheles species should be determined to assess the potential effect that mermithid parasitism may have on vector populations and malaria parasite transmission in order to define the utility of the mermithid as a biological control agent.

\section{Conclusions}

To our knowledge, this is the first report of mermithids from adult Anopheles mosquitoes in Senegal. Mermithids were present in $1.8 \%$ of the sampled adult Anopheles 
species in our study area. The mermithid was found in An. gambiae s.s., An. funestus, and An. rufipes which suggests parasite infection occurred in numerous, diverse mosquito larval habitats. Although maximum parsimony analysis determined the mermithid was closely related to S. spiculatus, several characteristics of the Senegalese mermithid were more similar to those more commonly associated with the Empidomermis genus, such as emergence from the adult mosquito. Future mermithid isolations of the Senegalese mermithid will hopefully allow for formal taxonomic identification, laboratory colonization, determination of life history traits and species specificity, and characterize its usefulness as a biological control agent.

\section{Competing interests}

The authors declare no competing interests.

\section{Authors' contributions}

KCK collected and processed specimens, analyzed data, and wrote the manuscript. MS collected specimens and wrote the manuscript. WBIV performed the phylogenetic analyses and wrote the manuscript. BDF collected specimens, provided reagents, and wrote the manuscript. All authors read and approved the final version of the manuscript.

\section{Acknowledgements}

We would like to thank the people of Ndebou, Boundoucondi, Nathia, and Damboucoye for allowing us to collect mosquitoes from their villages, Meg Gray for processing mosquito samples, and George Poinar Jr. for helpful discussion. This study was funded by grants R21Al079528 and R01Al094349 from the US National Institutes of Health.

Received: 16 May 2012 Accepted: 18 June 2012

Published: 28 June 2012

\section{References}

1. Poinar GO: Nematodes for biological control of insects. Boca Raton, FL: CRC Press; 1979.

2. Petersen JJ: Nematodes as biological control agents: Part I. Mermithidae Adv Parasit 1985, 24:307-346.

3. Poinar GO: Nematoda and Nematomorpha. In Ecology and Classification of North American Freshwater Invertebrates. 2nd edition. Edited by Thorp JH, Covich AP. San Diego, CA: Academic; 2001:255-295.

4. Blackmore MS: Mermithid parasitism of adult mosquitoes in Sweden. Am Midl Nat 1994, 132:192-198.

5. Kalucy EC: Parasitism of Anopheles annulipes Walker by a mermithid nematode. Mosquito News 1972, 32:582-585.

6. Blackmore MS, Berry RL, Foster WA, Walker ED, Wilmot TR, Craig GB: Records of mosquito-parasitic mermithid nematodes in the North-central United States. J Am Mosa Control Assoc 1993, 9:338-343.

7. Vythilingam I, Sidavong B, Seng TC, Phonemixay T, Phompida S, Krishnasamy M: First report of mermithid parasitism (Nematoda: Mermithidae) in mosquitoes (Diptera: Culicidae) from Lao PDR. Tropical Biomedicine 2005, 22:77-79.

8. Poinar GO, Camino NB: Strelkovimermis spiculatus n. sp. (Mermithidae: Nematoda) parasitizing Aedes albifasciatus Mac (Culicidae: Diptera) in Argentina. J Nematol 1986, 18:317-319.

9. Coz J: Contribution a l'etude du parasitisme des adultes d'Anopheles funestus par Gastromermis sp. (Mermithidae). Bull Soc Pathol Exot 1966, 59:881-889.

10. Poinar GO: Empidomermis cozii n. gen., n. sp. (Mermithidae: Nematoda) a parasite of Anopheles funestus (Culicidae: Diptera) in West Africa. Canadian J Zool 1977, 55:1475-1479.

11. Muspratt J: Observation on the larvae of tree-hole breeding Culicini (Diptera: Culicidae) and two of their parasites. J Entomol Soc South Africa 1945, 8:13-20
12. Obiamiwe BA, MacDonald WW: A new parasite of mosquitoes, Reesimermis muspratti sp. nov. (Nematoda: Mermithidae), with notes on Its life-cycle. Ann Trop Med Parasitol 1973, 67:439-444.

13. Platzer EG: Mermithid nematodes. J Am Mosa Control Assoc 2007, 23:58-64.

14. Juliano SA: Population dynamics. J Am Mosa Control Assoc 2007, 23:265-275

15. Petersen JJ: Biology of Octomyomermis muspratti, a parasite of mosquitoes as it relates to mass production. J Invertebr Pathol 1977, 30:155-159.

16. Petersen JJ, Chapman HC, Willis OR, Fukuda T: Release of Romanomermis culicivorax for control of Anopheles albimanus in El Salvador. II. Application of nematode. AmJTrop Med Hyg 1978, 27:1268-1273

17. Petersen JJ, Willis OR: Procedures for mass rearing of a mermithid parasite of mosquitoes. Mosquito News 1972, 32:226-230.

18. Levy R, Hertlein BC, Petersen JJ, Doggett DW, Miller TW: Aerial application of Romanomermis culicivorax (Mermithidae: Nematoda) to control Anopheles and Culex mosuqitoes in southwest Florida. Mosquito News 1979, 39:20-25.

19. Brown-Westerdahl B, Washino RK, Platzer EG: Successful establishment and subsequent recycling of Romanomermis culicivorax (Mermithidae: Nematoda) in a California rice field following post-parasite application. J Med Entomol 1982, 19:34-41.

20. Kerwin JL, Washino RK: Recycling of Romanomermis culicivorax (Mermithidae: Nematoda) in rice fields in California, USA. J Med Entomol 1985, 22:637-643.

21. Santamarina Mijares A: Actividad parasitaria de Romanomermis iyengari (Nematoda: Mermithidae) en criaderos naturales de larvas de mosquito. Miscellania Zoolaogica 1993, 17:59-65.

22. Santamarina Mijares A, Perez Pacheco R, Tomas Martinez SH, Canton LE, Ambrosio GF: The Romanomermis iyengari parasite for Anopheles pseudopunctipennis suppression in natural habitats in Oaxaca State, Mexico. Revista Panamericana de Salud Publica 1999, 5:23-28.

23. Santamarina Mijares A, Bellini AC: Mass production of Romanomermis iyengari (Nematoda: Mermithidae) applied to anopheline breeding sites in Boa Vista (Roraima), Brazil. Revista Panamericana de Salud Publica 2000, 7:155-161.

24. Willis $\mathrm{OR}$, Chapman $\mathrm{HC}$, Petersen Jj: Additional field testing of the mermithid parasite Romanomermis culicivorax against Anopheles albimanus in El Salvador. Mosquito News 1980, 40:71-73.

25. Santamarina Mijares A, Perez Pacheco R: Reduction of mosquito larval densities in natural sites after introduction of Romanomermis culicivorax (Nematoda: Mermithidae) in Cuba. J Med Entomol 1997, 34:1-4.

26. Rojas W, Northup J, Gallo O, Montoya AE, Montoya F, Restrepo M, Nimnich G, Arango $\mathrm{M}$, Echavarria $\mathrm{M}$ : Reduction of malaria prevalence after introduction of Romanomermis culicivorax (Mermithidae: Nematoda) in larval Anopheles habitats in Colombia. Bulletin of the W Health Org 1987, 65:331-337.

27. Sylla M, Kobylinski KC, Gray M, Chapman PL, Sarr MD, Rasgon JL, Foy BD: Mass drug administration of ivermectin in south-eastern Senegal reduces the survivorship of wild-caught, blood fed malaria vectors. Malar J 2010, 9:e365

28. Kobylinski KC, Sylla M, Chapman PL, Sarr MD, Foy BD: Short Report: Ivermectin Mass Drug Administration to Humans Disrupts Malaria Parasite Transmission in Senegalese Villages. AmJ Trop Med Hyg 2011, 85:3-5.

29. Diagne N, Fontenille D, Konate L, Faye O, Lamizana MT, Legros F, Molez JF, Trape JF: The Anopheles of Senegal - an annotated and illustrated check list. Bulletin De La Societe De Pathologie Exotique 1994, 87:267-277.

30. Scott JA, Brogdon WG, Collins FH: Identification of single specimens of the Anopheles gambiae complex by the polymerase chain reaction. AmJTrop Med Hyg 1993, 49:520-529.

31. Koekemoer LL, Kamau L, Hunt RH, Coetzee M: Cocktail polymerase chain reaction assay to identify members of the Anopheles funestus (Diptera: Culicidae) group. AmJTrop Med Hyg 2002, 66:804-811.

32. Thompson JD, Higgins DG, Gibson TJ, Clustal W: Improving the sensitivity of progressive multiple sequence alignment through sequence weighting, position-specific gap penalties and weight matrix choice. Nucleic Acids Res 1994, 22:4673-4680.

33. Gutell RR, Lee JC, Cannone JJ: The accuracy of ribosomal RNA comparative structure models. Curr Opin Struct Biol 2002, 12:301-310.

34. Gillespie JJ, McKenna CH, Yoder MJ, Gutell RR, Johnston JS, Kathirithamby J, Cognato Al: Assessing the odd secondary structural properties of nuclear 
small subunit ribosomal RNA sequences (18 S) of the twisted-wing parasites (Insecta: Strepsiptera). Insect Mol Biol 2005, 14:625-643.

35. Wilgenbusch JC, Swofford D: Inferring evolutionary trees with PAUP. Current Protocols in Bionformatics 2003,

36. Petersen JJ, Willis OR: Experimental release of a mermithid nematode to control Anopheles mosquitoes in Louisiana. Mosquito News 1974, 34:316-319.

37. Becnel JJ, Johnson MA: Pathogenicity tests on nine mosquito species and several non-target organisms with Strelkovimermis spiculatus (Nematoda: Mermithidae). J Nematol 1998, 30:411-414.

38. Achinelly ME, Micieli MV, Marti GA, Garcia JJ: Susceptibility of neotropical mosquito larvae (Diptera: Culicidae) and non-target aquatic organisms to the entomoparasitic nematode Strelkovimermis spiculatus Poinar \& Camino, 1986 (Nematoda: Mermithidae). Nematology 2004, 6:299-302.

39. Petersen JJ, Chapman HC: Parasitism of Anopheles mosquitoes by a Gastromermis sp. (Nematoda: Mermithidae) in southwestern Louisiana. Mosquito News 1970, 30:420-424.

40. Doucet MM, Laumond C, Bain O: Empidomermis riouxi nov sp (Nematoda: Mermithidae). Annales De Parasitologie Humaine Et Comparee 1979, 54:341-351.

41. Gaugler R, Wraight S, Molloy D: Bionomics of a mermithid parasitizing snow-pool Aedes spp. mosquitoes. Canadian J Zool 1984, 62:670-674.

42. St-Onge M, LaRue B, Charpentier G: A molecular revision of the taxonomic status of mermithid parasites of black flies from Quebec (Canada). $J$ Invertebr Pathol 2008, 98:299-306.

43. Gillies MT, de Meillon B: The Anophelinae of Africa south of the Sahara (Ethiopian zoogeographical region). Johannesburg: South African Institute of Medical Research Publication; 1968

44. Keiser J, Utzinger J, Castro MC, Smith TA, Tanner M, Singer BH: Urbanization in sub-Saharan Africa and implication for malaria control. AmJTrop Med Hyg 2004, 71:118-127.

45. Garrett-Jones C: Prognosis for interruption of malaria transmission through assessment of the mosquito's vectorial capacity. Nature 1964 204:1173-1175.

46. Davies JB, MCMahon JE, Beechgarwood P, Abdulai F: Does parasitism of Simulium damnosum by Mermithidae reduce the transmission of onchocerciasis? Trans Roy Soc Trop Med Hyg 1984, 78:424-425.

doi:10.1186/1756-3305-5-131

Cite this article as: Kobylinski et al:: Mermithid nematodes found in adult Anopheles from southeastern Senegal. Parasites \& Vectors 2012 5:131.

\section{Submit your next manuscript to BioMed Central and take full advantage of:}

- Convenient online submission

- Thorough peer review

- No space constraints or color figure charges

- Immediate publication on acceptance

- Inclusion in PubMed, CAS, Scopus and Google Scholar

- Research which is freely available for redistribution 